\title{
Computational method for simulating thermoset polymer curing and prediction of thermophysical properties
}

\author{
Jeffrey M. Sanders*a), Carla E. Estridge ${ }^{\text {b) }}$, Matthew B. Jackson ${ }^{\text {c) }}$, Thomas J.L. Mustard ${ }^{\text {a) }}$, Samuel \\ J. Tucker ${ }^{\text {b) }}$, David J. Giesen ${ }^{\text {a) }}$, Stephen Christensen ${ }^{\text {a) }}$, Andrea R. Browning ${ }^{\text {a) }}$ and Mathew D. \\ Halls ${ }^{\text {a) }}$ \\ a) Schrödinger Inc., New York, NY 10036 USA \\ b) The Boeing Company, St. Louis, MO 63134 USA \\ c) Solvay Composite Materials, Greenville, SC 29602 USA
}

\begin{abstract}
Thermoset polymers are an area of intense research due to their low cost, ease of processing, environmental resistance, and unique physical properties. The favorable properties of this class of polymers have many applications in aerospace, automotive, marine, and sports equipment industries. Molecular simulations of thermosets are frequently used to model formation of the polymer network, and to predict the thermomechanical properties. These simulations usually require custom algorithms that are not easily accessible to non-experts and not suited for high throughput screening. To address these issues, we have developed a robust cross-linking algorithm that can incorporate different types of chemistries and leverage GPU-enabled molecular dynamics simulations. Automated simulation analysis tools for cross-linking simulations are also presented. Using four well known epoxy/amine formulations as a foundational case study and benzoxazine as an example of how additional chemistries can be modeled, we demonstrate the power of the algorithm to accurately predict curing and thermophysical properties. These tools are able to streamline the thermoset simulation process, opening up avenues to in-silico high throughput screening for advanced material development.
\end{abstract}




\section{Introduction}

Thermoset and network polymers are an integral part of the composites industry and comprise $\sim 25 \%$ of the global plastics market [1]. Polymeric materials based on thermosets have a wide range of applications including aerospace, military, marine, and high performance consumer products. Composite materials with a specific range of physical properties are specified early on in the design process to enable product requirements to be met. Thermomechanical properties of thermosets are often the most important for industrial applications and are determined by both chemistry, molecular structure and network topology. Optimization of these properties for a specific application usually involves costly experiments and lengthy design projects.

Atomistic simulations of thermosets can reduce costs and accelerate the development cycle of new materials. For the case of thermoset polymers, modeling the evolution of the network structure formed during the cure cycle and prediction of thermomechanical performance can provide insight towards the structure-function relationship. With the advances in computational modeling due to technological breakthroughs in CPU and GPU-based computing, the need for costly experimental testing can be reduced through the use of simulations for determination of relevant thermoset properties. Previous work in the area of thermoset polymer simulations has demonstrated the ability to predict a variety of thermomechanical properties in agreement with experimental measurements ( 2 and references within). To date, simulation protocols developed to initialize thermoset polymer networks are generally specialized for a particular chemistry, sequestered within specific research groups, or require expert-level knowledge of the underlying simulation code to be useful. In this work, we present a crosslinking protocol that utilizes SMARTS (SMiles ARbitrary Target Specification) patterns [3] to specify the exact polymerization chemistry that takes place during the cross-linking process to allow for increased versatility/flexibility in application to a multiple chemistries..

The new cross-linking protocol is described with the simulation of the curing process of four well-known epoxy/amine systems and their thermophysical properties. To demonstrate the versatility of the algorithm in handling non-epoxy based chemistries, cross-linking of a benzoxazine system is also described. Using automated cross-linking analysis functionality, we are able to track the evolution of physical properties for each system as the cross-linking simulation proceeds. Once polymeric networks are formed, thermophysical simulations are used to predict properties including the glass transition temperature $\left(\mathrm{T}_{\mathrm{g}}\right)$ and the coefficient of thermal expansion. The ability to create polymer network structures and subsequently predict engineering relevant properties enables development of polymer systems that are optimized for targeted application.

\section{Results}


A cross-linking algorithm protocol was created that is versatile and capable of handling different types of chemistries and cross-linking procedures, depending on the system of interest. The details of the protocol are described using four epoxy-amine chemistries. To further demonstrate the utility of the tool, crosslinking of a benzoxazine system without any modification to the crosslinking protocol is also performed.

In the case of epoxy-based thermosets, amine-based hardeners are typically used to polymerize the matrix. The epoxide ring of the epoxy molecule reacts with primary and secondary amines via a ring opening process. Primary amines will react with the alpha carbon of the epoxide ring, leading to the formation of a secondary amine and a hydroxyl group (Figure 1) [4]. To define this chemical reaction in a molecular model SMARTS patterns are utilized to define the two bonds to be broken with a high level of specificity. For epoxy/amine mixtures, the user can define both primary and secondary amines together or as separate reactions. Several studies have shown that reaction rates of primary versus secondary amines are different, but there is some disagreement to the relative reaction ratio [5-7]. Multiple reactions can also be defined, including those initiated by hydroxyl groups, but are not the subject of this work. The majority of molecular simulation reports on thermosets do not incorporate different reactive rates, and in some cases require opening of the epoxide rings prior to forming the crosslinking bond [8-13]. These intermediate molecules are not necessarily representative of the crosslinking reaction, and can be avoided by properly defining SMARTS patterns for the $\mathrm{N}-\mathrm{H}$ and $\mathrm{C}-\mathrm{O}$ bonds of the two species. The reaction coordinates can then be defined by the distance between the two reactive bonds. The distance criteria can be set to define a minimum distance required for bond formation, along with a cutoff distance. The cutoff distance can greatly affect the topology of the polymer network. As the equilibrium bond length of an $\mathrm{N}-\mathrm{C}$ bond is $1.41 \AA$, the cutoff distance should be 3-4 times this length to prevent unnecessarily high energy bonds from being created [13]. Our cross-linking algorithm allows the distance criteria, including the incremental step size, to be controlled by the user and can be adjusted based on the reaction(s) being modeled but suggested values discussed later in this work allow for reasonable structures to be obtained.

Once the chemistry and distance criteria are defined, the details of the cross-linking procedure is set (Figure 2). The process of forming multiple, simultaneous crosslinking bonds influences the resulting network topology and thermomechanical properties [14]. By gradually introducing new bonds and equilibrating the intermediate structures, the simulation is able to mimic the curing the process. Several parameters in the crosslinking are adjustable based on the specific system. First, the number of simultaneous cross-links per iteration is defined. A crosslinking iteration can be defined as the number of simultaneous cross links formed in the simulation, followed by force field typing and a short MD equilibration stage. Crosslinking algorithms often require partial charge fitting and subsequent equilibration when generating the force field terms for the new crosslinked bonds [9]. These methods are diverse, not well 
documented and computationally expensive [9, 13-17]. Utilizing the OPLS2005 force field, a robust force field developed for condensed phase simulations, automatic force field typing can be applied, avoiding the aforementioned charge equilibration process [18-21].

In addition to controlling the number of crosslinks formed per iteration, the target cure percentage can also be defined. As the system becomes highly crosslinked, above $80 \%$ for epoxy-amine thermosets, the number of available reactive groups decreases and the steric constraints increases substantially and may never fall within the distance cutoff. Depending on the property of interest to the user, the final crosslink saturation percentage can be adjusted accordingly. To limit the computational time required for a crosslinking simulation with a high target saturation, a maximum number of unproductive crosslinking iterations is set. An unproductive crosslinking iteration occurs when no crosslinks can be made with the user-defined distance criteria. Following both successful and unsuccessful crosslinking iterations the MD equilibration protocol is performed, allowing fluctuations in the system to occur that may allow for crosslinking in subsequent iterations.

The MD equilibration step in each iteration is designed to minimize high energy states from newly formed bonds and adjust for the changing topology of the system. The simulation time, temperature, and integration time step can be independently adjusted for the equilibration of the system curing the curing process. One common system property that is often used to measure MD simulation stability is density [22]. If the density of the system is rapidly fluctuating, longer simulation times may be necessary for equilibration. Previous crosslinking algorithms do not adjust for longer simulation times on the fly, which may lead to poorly equilibrated structures or insufficient sampling required to reach a high degree of crosslinking saturation [2 and references within]. To address this issue a density convergence criteria was enforced allowing the density to stabilize through multiple MD equilibration stages. The density convergence is determined by comparing the average and standard deviation over the last $20 \%$ of the simulation. If the standard deviation is not less than $5 \%$ of the average, it is not considered converged. The process is repeated until the target saturation is reached, or the maximum number of unproductive cross-linking attempts (with MD equilibration) has been reached. By utilizing GPU acceleration, computational times required for these equilibration stages is reduced. This capability helps accelerate the equilibration stage, which can be rate limiting depending on the size of the system, and produce more equilibrated thermoset structures.

Modeling the curing process of epoxy/amine systems To validate the utility of the cross-linking algorithm we performed cross-linking simulations on four well-known epoxy/amine systems (Figure 3). These systems have either been experimentally validated and/or have been modeled previously. Before building a highly cross-linked network, we first generated a set of amorphous systems for all four mixtures. Systems were built so that the reactive group 
stoichiometry was 1:1. Diglycidyl ether of bisphenol A (DGEBA) and diglycidyl ether of bisphenol F (DGEBF) both have a functionality of two, and 3,3-Diamino Diphenyl Sulfone (3,3-DDS) and the 4,4-DDS isomer have a functionality of four and tris(4-aminophenyl)amine (TAPA) six, so the relative monomer ratios were adjusted to ensure all systems have a one to one ratio of epoxide to amine functional groups. It is important to point out that with molecular models of thermoset polymers, it is difficult to capture the entire network architecture topology unless the system is sufficiently large to account for finite size effects. A recent study indicated that relatively small system sizes (less than $5 \mathrm{k}$ atoms) can decrease the accuracy of thermo-mechanical property predictions like the glass transition temperature [23]. To avoid this issue, we built systems in system sizes close to or exceeding 19k atoms. The system size used in this study was determined to be sufficient to study the majority of cases described by performing simulations at a smaller simulation size (close to or exceeding 10K) and confirming that the conclusions remained consistent. A total of 10 replicates were simulated for all four systems. As the crosslinking and thermophysical properties protocols both utilize GPU enabled MD, the simulation times are tractable to obtain results on multiple systems and replicates within a short period of time.

After relaxing each system as described in Simulation Procedures section, a final stage with a high temperature ( $800 \mathrm{~K}$ ) was run for $5 \mathrm{~ns}$ to prepare for the crosslinking simulation to converge the density within 5\% at the temperature determined to be most appropriate for cross-linking these systems. The high temperature is necessary during crosslinking to reduce the bond energy and also to allow enhanced sampling of the reactive groups due to increased molecular mobility to form more crosslinks. Simulation temperatures for crosslinking thermosets are frequently higher than those used in experiment and can affect the final cure saturation [2]. To quantify this effect, we chose one system and ran crosslinking simulations over a range of temperatures. The equilibrated DGEBA/3,3-DDS system was crosslinking at $100 \mathrm{~K}$ to $900 \mathrm{~K}$ at $100 \mathrm{~K}$ intervals (Figure 4a). Above $600 \mathrm{~K}$, the system was able to achieve a final saturation of $90 \%$ or greater. At temperatures above $700 \mathrm{~K}$, the final cure was above $96 \%$. This lead us to choose $800 \mathrm{~K}$ as the crosslinking temperature for the other three systems. The resulting structures were all cured above $95 \%$, a higher percentage than most systems previously studied [2 and references within]. This suggests that high cure percentages can be achieved with high temperature cross-linking.

At a molecular level, the increasing cure with temperature can be explained by increased sampling of phase space, allowing amine and epoxy groups to come within reactive distances more frequently. To minimize the steric clashes further, we limited the maximum number of cross-links formed in each iteration to $1-2 \%$ of the effective curing saturation and set the maximum cross-linking distance between the amine nitrogen and the oxirane alpha carbon to 5 $\AA$. Forming bonds between reaction sites above this cutoff exceeds the normal bond distance by 
4 times, leading to high energy configurations and system instability [10].

We next sought to determine the optimal simulation time required for equilibration at each iteration by asking what affect equilibration times have on the final cure percentage. Using the DGEBA/3,3-DDS system, we crosslinked the system with varying equilibration simulation times. Equilibration times of 25 ps or greater per iteration produced highly cross-linked systems above $95 \%$ final saturation (Figure $4 b$ ). The final cure did not change significantly with simulation times longer than 50ps. Optimizing these parameters is important not only for obtaining high cure percentages, but also balancing simulation time. If a user wishes to use this tool in a high throughput screening method to explore more chemical space with either the resin or reactants, longer simulations are not necessarily beneficial. Resulting thermoset networks from crosslinking simulations, and the quality of each result, need to be analyzed before subjecting them to thermophysical prediction protocols.

Analysis of MD simulations frequently requires custom scripting, particularly for complicated workflows like crosslinking. We developed automated analyses that can speed up the process, allowing researchers to efficiently analyze the data. The analyses were developed to present the data in a way that gives the user insight on the physical changes occurring during the cure process. For epoxy-based resin systems, one important physical change that occurs while curing is volume shrinkage. As more crosslinks are formed throughout the system, the distance between monomers and oligomers decreases. This effect has been observed experimentally and is an important factor when designing new formulations [24-25]. While volume shrinkage is measurable via experiment, the effects of chemical shrinkage depend on several factors including thermal expansion and final cure percentage. These properties typically depend on the molecular structure and are not easily accessible with experimental methods. Simulation methods, however, can measure exact changes in chemical structures and bulk physical properties of the molecular system.

An analysis feature was designed to gather not only volume information, but also other physical parameters and crosslinking statistics at each iteration. The changes in volume were collected for the four representative epoxy systems (Figure 5). For the four systems, volume decreased linearly as the cure simulation progressed with final volume shrinkage ranging from $1 \%$ to $2 \%$ for structures cooled to $800 \mathrm{~K}$. This linear relationship between reduction of volume and increasing cure is in agreement with experiment [26-28]. The total shrinkage from curing simulations has been reported for several systems, ranging from 4-12\% [22, 29-30]. While our recorded shrinkage is lower than experimental value, behavior of the CTE as the crosslinking progresses is consistent to reported behavior for highly crosslinked systems using both experiment and other molecular simulations $[31,32]$. In the experimental study, the maximum volume shrinkage for the thermoset occurred after gelation and depended on the overall crosslinking density. The authors attributed this effect to entropic changes through a dropoff of 
molecular packing efficiency in a highly cured polymeric network. Based on the experimental and simulation studies, we can conclude that the lower final volume shrinkage values reported here are a result of the crosslinker's ability to achieve high final crosslinking cure percentages, $>95 \%$, reducing ability of the network to pack efficiency and may more accurately reflect the cure process itself. We do not attempt to explain the detailed molecular basis of this observation, but future work can aim to study the morphology of thermoset networks and the thermodynamic contributions, both enthalpic and entropic, to curing.

Gelation is a distinguishing feature of thermoset polymers as it represents the point in the cure cycle where the largest molecule percolates through the entire sample [2]. As a thermoset polymer approaches the gel point, its viscosity approaches infinity, and elastic properties of the system change dramatically. Gel points are often difficult to estimate experimentally using either dynamic mechanical analysis (DMA) or dynamic rheology. Theoretical gel points can be calculated, requiring only the functionality of the prepolymer and the crosslinking agent and their stereochemistry. Flory derived this equation (1) as:

$r p^{2}=\frac{1}{(f-1)(g-1)}$

where $\mathrm{r}$ is the stoichiometric ratio, $p$ is the gel point, $f$ is the functionality of the epoxy and $g$ the functionality of the crosslinking agent [33]. In molecular simulations, the gel point can be approximated using the weight averaged reduced molecular weights of the largest molecules as the system cures [34]. Using this method, we approximated the the gel points from each simulation and found them to be within 5-7\% of their respective theoretical values (Figure 6). It is important to point out that both of these methods assume perfect reaction stoichiometry. Off-stoichiometric effects on physical property prediction can be studied by altering the epoxy to amine ratios.

While bulk physical properties of the system are important, tracking the actual reactive groups can provide insight into the morphology of the thermoset polymer. Reactive group concentrations, are often measured experimentally using near IR (NIR) spectroscopy. NIR is able to identify all the reactive groups (primary and secondary amines along with $\mathrm{C}-\mathrm{H}$ epoxy bonds) and their products. While this method has been useful for quantifying amine reactivity, it is often difficult to track all chemical functionality of interest [35]. Using SMARTS patterns, any chemical substructure and its concentration can be identified during the cure cycle. For the DGEBA/3,3-DDS system, we tracked SMARTS patterns corresponding to primary and secondary amines, along with hydroxyl formation (Figure 7a). The general shapes of the curves for primary and secondary amines are in agreement with NIR data for other epoxy/amine systems [35]. In addition to quantifying the reactive groups and their products, SMARTS can be 
used to provide some insight to morphology, specifically the location of unreactive groups or dangling ends. Figure $7 \mathrm{~b}$ shows the location of the unreacted amine and epoxide groups in the simulation cell after curing to $98 \%$. This type of analysis can be extended to study large structural motifs created during crosslinking, providing a molecular level picture of morphological changes as the polymer network is formed.

\section{Modeling the curing process of benzoxazine system}

To demonstrate the flexibility of cross-linking algorithm utilizing reactions defined SMARTS patterns, crosslinking of a benzoxazine system was performed. The same cross-linking algorithm as described in the epoxy-amine system was applied to the chain growth polymerization of a bisphenol-A based benzoxazine. Though the chemical details for this thermoset differ from the epoxy-amine case described in the previous section, the definition of cross-linking via two breaking bonds and two forming bonds allows for the application of the same cross-linking algorithm.

The cross-linking reaction in this demonstration study was defined as ring opening followed by monomer connection (Figure 8) [36, 37]. A crosslinking of a single chemistry was performed at cure temperature $(500 \mathrm{~K})$ selected to best match the typical cure temperature [36]. There is known dependency of the properties such as $T_{g}$ of the final thermoset on the cure and the purity of the benzoxazine, therefore a more thorough study of benzoxazine simulation is necessary to fully capture the effects of variation in network formation. The reduced molecular weight as well as change in volume and density are shown in Figure 8 and demonstrate the cure progression in this example case. More detailed studies of benzoxazine thermosets are reserved for future publication.

\section{Glass transition temperature and volumetric coefficient of thermal expansion prediction}

One of the most attractive properties of thermoset polymers is their high glass transition temperature [10]. Several experimental methods are available to estimate $\mathrm{T}_{\mathrm{g}}$ and are widely used by engineers/researchers. Time scales of these experiments are generally much longer, on the order of minutes to hours, than accessible by even the most efficient MD codes. Given the cooling rate dependence of $\mathrm{T}_{\mathrm{g}}$, exact quantitative prediction from MD cooling simulations is not possible yet [4]. Despite the difference in time scales, several studies have shown molecular simulations do have predictive power when estimating $T_{g}$ values [10-13]. Typically, most MD simulations overestimate the $\mathrm{T}_{\mathrm{g}}$ by $\sim 30 \mathrm{~K}$ due to the fast cooling rates [2]. Using the four highly crosslinked structures, we performed annealing simulations using the thermophysical properties workflow. Starting at $800 \mathrm{~K}$, each system was cooled to $100 \mathrm{~K}$ at a rate of $0.5 \mathrm{~ns}$ per kelvin with steps of $10 \mathrm{~K}$. At each step, the density convergence criteria of $5 \%$ was applied to each $5 \mathrm{~ns}$ simulation at a given temperature, ensuring each density measurement was accurate across the 
range of temperatures. This rate was deemed sufficient for this study by running of a slower rate of $2 \mathrm{~ns}$ per kelvin (data not shown). The comparison in $\mathrm{T}_{\mathrm{g}}$ of the different systems was found to be similar between the $2 \mathrm{~ns} / \mathrm{K}$ versus $0.5 \mathrm{~ns} / \mathrm{K}$. The total simulation time at the rate of $2 \mathrm{~ns} / \mathrm{K}$ for each system was $1.44 \mu \mathrm{s}$, representing significantly longer simulations than previous annealing simulations to estimate $T_{g}$ [38-39]. These simulations required approximately one week of computational time on a single GPU even with the comparably slow rate.

Estimation of $\mathrm{T}_{\mathrm{g}}$ itself from molecular simulations is not a straightforward process as finite size effects and shorter time scales introduce noise, drastically affect the fitting process. Fitting procedures, including the popular bilinear method, rely on subjective definitions of the two regions and do not utilize all the data generated [40]. Recently, Patrone et al. proposed a global nonlinear fitting method that makes use of an entire simulated annealing dataset and methods to estimate the uncertainty of their fitting method [22]. We adopted the hyperbola fit method from that work to estimate $\mathrm{T}_{\mathrm{g}}$ from the density data as a function of temperature. Hyperbolic fits of each dataset produced $\mathrm{T}_{\mathrm{g}}$ values based on the hyperbola center (Figure 9 and Table 1). The $95 \%$ confidence interval predicted from the hyperbola fit was also calculated to quantify the uncertainty between the 10 replicates of each system. The uncertainty ranged from 5-26K (1-5.4\% of simulated $\mathrm{T}_{\mathrm{g}}$ ) and demonstrates that the crosslink and $\mathrm{T}_{\mathrm{g}}$ method provide consistent result across random initial conditions.

In the cases with the 4 functional amines (3,3-DDS and 4,4-DDS), the trend in $T_{g}$ is reproduced compared to experiment. The absolute value of the deviation between the simulation and experiments ranges from $7-17 \mathrm{~K}$ and when factoring in the difference in time scales between experiment and microsecond MD simulations, these values are in excellent agreement and consistent with previously reported computational studies [2 and references within]. The 6-functional cured system (DGEBA-TAPA) shows a larger deviation from experiments and does not match the trend found in the other systems. The source of this discrepancy may be the system size. Future studies are needed to fully capture this potential impact.

In addition to $T_{g}$, the coefficient of thermal expansion (CTE) is another critical property in material design that can be predicted using molecular simulation [16]. The volumetric coefficient of thermal expansion (a) can be defined as:

$\alpha=\frac{1}{V_{0}}\left(\frac{\partial V}{\partial T}\right)_{P}$

where $V$ is the volume of the simulation cell at a given temperature $\mathrm{T}$ and $V_{0}$ is the volume of the simulation cell at a reference temperature [2]. This value can be calculated at any temperature above and below the $\mathrm{T}_{\mathrm{g}}$. For each system, we calculated CTE values at $300 \mathrm{~K}$ and $600 \mathrm{~K}$, below and above their $\mathrm{T}_{\mathrm{g}}$ values, respectively. Experimental data on three of the systems, 
DGEBA/DEGBF crosslinked with either 3,3-DDS or 4,4-DDS, were provided by [41] (Table 2). For the DEGBF/3,3-DDS system, [43] reported a linear glassy CTE of $57.2 \mu \mathrm{e} / \mathrm{K}$ from TMA experiments and $55.7 \mu \mathrm{e} / \mathrm{K}$ from their MD simulations while we obtained a value of $49.5+/-1.7$ $\mu \mathrm{e} / \mathrm{K}$ at $300 \mathrm{~K}$ averaged over the 10 replicates. For the rubbery CTE, they reported a linear CTE of $170.8 \mu \mathrm{e} / \mathrm{K}$ from experiment with our simulations estimating the CTE of $188.7+/-2.1$ at $600 \mathrm{~K}$. Li and Strachan analyzed the literature for predicted CTE values and found predicted values deviated from experiment by up to $40 \%$ [2]. Our predicted values for the three systems (no CTE data was available for DGEBA/TAPA) deviated 13-26\% from experimental values. The magnitude ranking of CTE for the three systems was also reproduced. The overall increased accuracy of our results compared to general simulation literature suggest that CTE prediction is possible with the combination of the discussed cross-linking method and slow annealing for property calculations.

\section{Discussion}

The need for a flexible, user-friendly cross-linking method to generate equilibrated atomistic models of highly cross-linked thermosets motivated the development of a general, robust, and efficient polymer cross-linking protocol. While previous methods require custom code, long simulation times and are difficult to reproduce, this method is intended to be accessible to the general modeling and materials engineering community and generate accurate results faster. To test the utility of the crosslinking algorithm, we took four epoxy/amine formulations and optimized the simulation parameters to generate highly crosslinked structures, well above $90 \%$. In order to judge the quality of a crosslinked polymer model, automated analysis functionality was designed to monitor the physical and morphological changes that occur to the system during the simulation. Utilizing SMARTS theory, we are able to not only flexibly designate the reactive bonds for crosslinking but also analyze relevant structural motifs as curing progresses. By performing thermophysical properties calculations on the crosslinked systems, we are able to compare $\mathrm{T}_{\mathrm{g}}$ predictions with experiment. The agreement between the results generated here and previous experimental works demonstrates the accuracy of the OPLS2005 force field while simultaneously leveraging GPU accelerated MD simulations. This can also be extended to predicting mechanical response of thermoset polymer networks, further increasing the modeling power to predict thermomechanical properties. While epoxy/amine systems were used in this study, any crosslinking chemistry can be simulated were the bonds broken and formed during the reaction are known. Systems where crosslinking contains multiple competing or sequential reactions steps/reactions, previously intractable with existing algorithms, can also be simulated with this method and will be explored in future work. 


\section{Methods}

Molecular models and Molecular Dynamics equilibration All model building and molecular dynamics simulations were performed using the Schrödinger Materials Science Suite version 2016-4 [42]. The molecular models of DGEBA, DGEBF, 3,3-DDS, 4,4-DDS, and TAPA were built using the 2-D sketcher, and 3-D conformations were generated with the OPLS2005 force field [18-19]. Model systems of epoxy and amine molecules, with a 1 to 1 reactive group stoichiometry, were built using the Disordered System Builder at a low density $(<0.5 \mathrm{~g} / \mathrm{cc})$. The resulting amorphous system was equilibrated using the Multistage Simulation Workflow panel with the compression protocol (a series of short MD relaxations and a compressive NPT MD simulation performed at high pressure to increase the density) and then further equilibrated at $800 \mathrm{~K}$ for 5ns with a NPT ensemble using the Nosé-Hoover thermostat [43] and Martyna-Tobias-Klein barostat [44]. All simulations were analyzed using the Materials Science MD analysis functionality. All MD simulations were performed using the Desmond Molecular Dynamics Engine [45, 46, 47].

Crosslinking polymers with multiple reactions The curing stage of the thermoset was modeled using the Crosslink Polymers driver. Chemical reactions are set up in a stepwise manner using a distance-based criteria. The bonds broken and formed during the simulation are defined using SMARTS patterns [3]. The following SMARTS patterns were used: "N[H]" for primary and secondary amines and " $[\mathrm{C} ; \mathrm{r} 3 ; \mathrm{H} 2] \mathrm{O}$ " for the carbon-oxygen bond in the epoxide ring. The starting search radius for reactive bonds was set to $3 \AA$ while maximum search distance was set to $5 \AA$ with a 0.5 A step. The number of cross-links performed at each iteration was limited to $1-2 \%$ of all amine/epoxy reactions for each system. After the new bonds are formed at each iteration, a 50ps multistep relaxation protocol is applied to relax the system. The simulation time was determined by cross-linking one system, DGEBA/3,3-DDS using the following values: 5ps, 10ps, 25ps, 50ps, 100ps, 250ps and 400ps. To determine the optimum temperature, the DGEBA/3,3-DDS system, was cross-linked at the following temperature range: $100-900 \mathrm{~K}$ with data collected at each $100 \mathrm{~K}$ iterations. The four systems were then cross-linked at $800 \mathrm{~K}$ with a 50ps MD equilibration time. All crosslinking analysis was performed using the crosslink polymers analysis module. 
Glass transition temperature prediction Glass transition temperatures of the cross-linked systems were calculated using the thermophysical properties driver. The systems were cooled from $800 \mathrm{~K}$ to $100 \mathrm{~K}$ at a rate of $0.5 \mathrm{~ns}$ per Kelvin. At each temperature, a density convergence criterion was applied to within $5 \%$ of the "running average". For the systems studied in this work, no data points required additional simulation time to converge the density. The $\mathrm{T}_{\mathrm{g}}$ was determined by performing a hyperbola fit to the density versus temperature data, a method discussed in [22]. The volumetric coefficient of thermal expansion was estimated by linear fits to the glass and rubbery regions of each dataset. CTE values were reported at both $300 \mathrm{~K}$ and $600 \mathrm{~K}$. 


\section{References}

1. Caliendo, H. Roundup of composites market outlooks and forecasts. CompositesWorld (2015). URL: http://www.compositesworld.com/news/roundup-of-composites-industry-outlook-

2. Li, C. \& Strachan, A. Molecular scale simulations on thermoset polymers: A review. J. Polym. Sci. Part B Polym. Phys. 53, 103-122 (2015)

3. SMARTS - A Language for Describing Molecular Patterns; http://www.daylight.com/dayhtml/doc/theory/theory.smarts.html

4. May, Clayton, ed. Epoxy resins: chemistry and technology. CRC press, 1987.

5. Matějka, Libor. "Amine cured epoxide networks: Formation, structure, and properties." Macromolecules 33.10 (2000): 3611-3619.

6. Wang, Xiaorong, and John K. Gillham. "Competitive primary amine/epoxy and secondary amine/epoxy reactions: Effect on the isothermal time-to-vitrify." Journal of applied polymer science 43.12 (1991): 2267-2277.

7. Vogel, Wouter, et al. "Synthesis and characterisation of new sulphur-containing epoxy networks." High Performance Polymers 26.4 (2014): 420-435.

8. Jang, Changwoon, et al. "Relative reactivity volume criterion for cross-linking: Application to vinyl ester resin molecular dynamics simulations." Macromolecules 45.11 (2012): 4876-4885.

9. Demir, Baris, and Tiffany R. Walsh. "A robust and reproducible procedure for cross-linking thermoset polymers using molecular simulation." Soft matter 12.8 (2016): 2453-2464.

10. Li, Chunyu, et al. "Molecular dynamics simulations and experimental studies of the thermomechanical response of an epoxy thermoset polymer." Polymer 53.19 (2012): 4222-4230.

11. Shenogina, Natalia B., et al. "Molecular modeling approach to prediction of thermo-mechanical behavior of thermoset polymer networks." Macromolecules 45.12 (2012): 5307-5315.

12. Kang, Jingtian, et al. "Nanoscale crosslinking in thermoset polymers: a molecular dynamics study." Physical Chemistry Chemical Physics 17.25 (2015): 16519-16524.

13. Li, Chunyu, Eric Coons, and Alejandro Strachan. "Material property prediction of thermoset polymers by molecular dynamics simulations." Acta Mechanica 225.4-5 (2014): 1187.

14. Li, Chunyu, and Alejandro Strachan. "Molecular simulations of crosslinking process of thermosetting polymers." Polymer 51.25 (2010): 6058-6070.

15. Li, Chunyu, and Alejandro Strachan. "Molecular dynamics predictions of thermal and 
mechanical properties of thermoset polymer EPON862/DETDA." Polymer 52.13 (2011): 2920-2928.

16. Li, Chunyu, et al. "Atomistic simulations on multilayer graphene reinforced epoxy composites." Composites Part A: Applied Science and Manufacturing 43.8 (2012): 1293-1300.

17. Li, Chunyu, Eugenio Jaramillo, and Alejandro Strachan. "Molecular dynamics simulations on cyclic deformation of an epoxy thermoset." Polymer 54.2 (2013): 881-890.

18. Jorgensen, William L., and Julian Tirado-Rives. "The OPLS [optimized potentials for liquid simulations] potential functions for proteins, energy minimizations for crystals of cyclic peptides and crambin." Journal of the American Chemical Society 110.6 (1988): 1657-1666.

19. Jorgensen, William L., David S. Maxwell, and Julian Tirado-Rives. "Development and testing of the OPLS all-atom force field on conformational energetics and properties of organic liquids." Journal of the American Chemical Society 118.45 (1996): 11225-11236.

20. Shivakumar, Devleena, et al. "Prediction of absolute solvation free energies using molecular dynamics free energy perturbation and the OPLS force field." Journal of chemical theory and computation 6.5 (2010): 1509-1519.

21. Banks, J. L. et al. "Integrated modeling program, applied chemical theory (IMPACT)." $J$. Comput. Chem. 26 (2005): 1752-1780.

22. Varshney, Vikas, et al. "A molecular dynamics study of epoxy-based networks: cross-linking procedure and prediction of molecular and material properties." Macromolecules 41.18 (2008): 6837-6842.

23. Patrone, Paul N., et al. "Uncertainty quantification in molecular dynamics studies of the glass transition temperature." Polymer 87 (2016): 246-259.

24. Rogers, K. F., et al. "The thermal expansion of carbon fibre-reinforced plastics." Journal of Materials Science 12.4 (1977): 718-734.

25. Lubin, George. Handbook of composites. Springer Science \& Business Media, 2013.

26. Zarrelli, Mauro, Alexandros A. Skordos, and Ivana K. Partridge. "Investigation of cure induced shrinkage in unreinforced epoxy resin." Plastics, rubber and composites 31.9 (2002): 377-384.

27. Kravchenko, Oleksandr G., Sergii G. Kravchenko, and R. Byron Pipes. "Chemical and thermal shrinkage in thermosetting prepreg." Composites Part A: Applied Science and Manufacturing 80 (2016): 72-81.

28. Nawab, Yasir, Salma Shahid, Nicolas Boyard, and Frédéric Jacquemin. "Chemical shrinkage characterization techniques for thermoset resins and associated composites." Journal of Materials Science 48.16 (2013): 5387-5409. 
29. Yarovsky, Irene, and Evan Evans. "Computer simulation of structure and properties of crosslinked polymers: application to epoxy resins." Polymer 43.3 (2002): 963-969.

30. Yang, Shaorui, and Jianmin Qu. "Computing thermomechanical properties of crosslinked epoxy by molecular dynamic simulations." Polymer 53.21 (2012): 4806-4817.

31. Bandyopadhyay, Ananyo, et al. "Molecular modeling of crosslinked epoxy polymers: The effect of crosslink density on thermomechanical properties." Polymer 52.11 (2011): 2445-2452.

32. Wang, Xiaorong, and Victor J. Foltz. "Crosslinking induced volume expansion in the glass state." Polymer 47.14 (2006): 5090-5096.

33. Flory, Paul J. Principles of polymer chemistry. Cornell University Press, 1953.

34. Cheng, Kuo-Chung, and Wen-Yen Chiu. "Monte-Carlo Simulation of Polymer Network Formation with Complex Chemical-Reaction Mechanism-Kinetic Approach on Curing of Epoxides with Amines." Macromolecules 27.12 (1994): 3406-3414.

35. González, María González, Juan Baselga, and Juan Carlos Cabanelas. Applications of FTIR on epoxy resins-identification, monitoring the curing process, phase separation and water uptake. INTECH Open Access Publisher, 2012.

36. Ishida, Hatsuo and Tarek Agag. "Handbook of Benzoxazine Resins." Elsevier (2011).

37. Saiev, Shamil, Leila Bonnaud, Philippe Dubois, David Beljonne, and Roberto Lazzaroni. "Modeling the formation and thermomechanical properties of polybenzoxazine thermosets." Polymer Chemistry 8.38 (2017): 5988-5999.

38. Buchholz, Joachim, et al. "Cooling rate dependence of the glass transition temperature of polymer melts: Molecular dynamics study." The Journal of chemical physics 117.15 (2002): 7364-7372.

39. Barrat, Jean-Louis, and Michael L. Klein. "Molecular dynamics simulations of supercooled liquids near the glass transition." Annual Review of Physical Chemistry 42.1 (1991): 23-53.

40. Forrest, J. A., et al. "Effect of free surfaces on the glass transition temperature of thin polymer films." Physical review letters 77.10 (1996): 2002.

41. Knowles, Kyler. "Effect of chain rigidity on network architecture and deformation behavior of glassy polymer networks." PhD dissertation, University of Southern Mississippi, Department of Polymers and High Performance Materials, Spring (2017).

42. Materials Science Suite 2016-4, Schrödinger, LLC, New York, NY, 2016.

43. Hoover, William. "Canonical dynamics: Equilibrium phase-space distributions." Physical Review A 33.3 (1985): 1695.

44. Martyna, Glenn, Douglas Tobias, and Michael Klein. "Constant pressure molecular dynamics algorithms." Journal of Chemical Physics 101.5 (1994): 4177-4189.

45. Schrödinger Release 2016-4: Desmond Molecular Dynamics System, D. E. Shaw 
Research, New York, NY, 2016

46. Maestro-Desmond Interoperability Tools, Schrödinger, New York, NY, 2016.

47. Kevin J. Bowers, Edmond Chow, Huafeng Xu, Ron O. Dror, Michael P. Eastwood, Brent A. Gregersen, John L. Klepeis, Istvan Kolossvary, Mark A. Moraes, Federico D. Sacerdoti, John K. Salmon, Yibing Shan, and David E. Shaw, "Scalable Algorithms for Molecular Dynamics Simulations on Commodity Clusters", Proceedings of the ACM/IEEE Conference on Supercomputing (SC06), Tampa, Florida, November 11-17, 2006.

48. Su, Wen-Chiung et al. "Development of a cross-linked epoxy resin with flame-retardant properties.” Patent US7981980 B2. Jul 19, 2011. 


\section{Acknowledgements}

The authors would like to acknowledge Paul Patrone of National Institute of Standards and Technology, Gaithersburg, MD for many insightful conversations on quantification of uncertainty in $\mathrm{T}_{\mathrm{g}}$ simulations and the interpretation of uncertainty results.

\section{Author Contributions}

JMS performed all simulations and wrote the main manuscript text. CEE prepared figures 5-7 and performed uncertainty quantification calculations. CEE, MBJ, and SJT provided input on crosslinking procedure. JMS, TJLM, DJG, and SC implemented the crosslinking procedure in Schrödinger Materials Science Suite. ARB, and MDH provided oversight for the study. All authors reviewed the manuscript.

\section{Additional Information}

\section{Competing financial interests}

Drs. Sanders, Mustard, Giesen, Browning, and Halls are employed by Schrödinger Inc. Mr. Christensen is a consultant of Schrödinger Inc. 


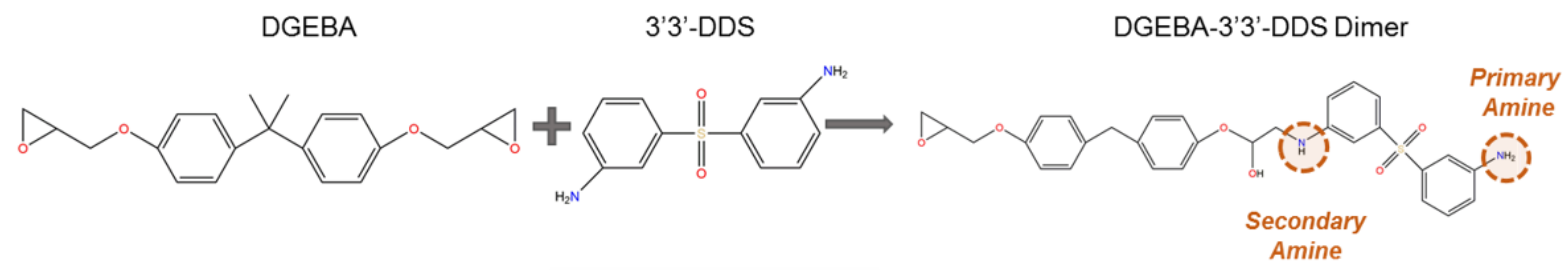

Figure 1: Representative Epoxy-amine reaction. The initial reaction occurs between a primary amine of $3^{\prime} 3^{\prime}$-DDS and one of the epoxide rings of DGEBA. After the first crosslinking reaction, the dimer created now has one secondary amine, one primary amine and one epoxide group that may take place in subsequent crosslinking reactions. 


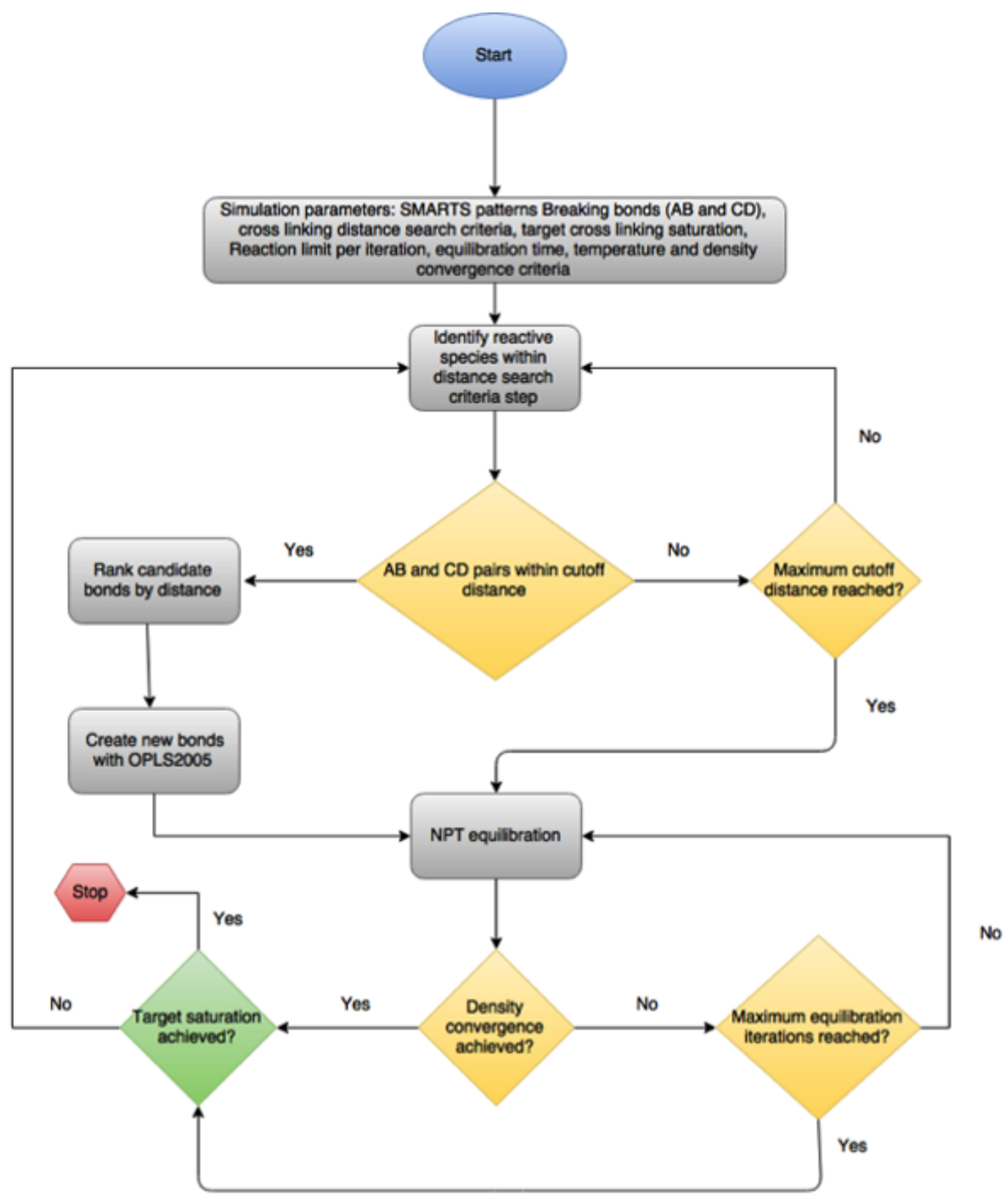

Figure 2: Cross-linking flowchart. The input parameters along with the amorphous system are used by the crosslink driver to determine the reactive groups, distance search criteria, and number of cross-links to be performed at each iteration. After the new bonds are formed and the force field typing is finished, the system is subject to a MD simulation. If a density convergence criteria is imposed by the user, the driver will attempt to run MD until the convergence criteria is met. Once the MD steps are finished, a single iteration is considered completed. If the target saturation has not been met, the process is repeated until achieved or the maximum number of unsuccessful cross-linking attempts are reached. 
1) DGEBA

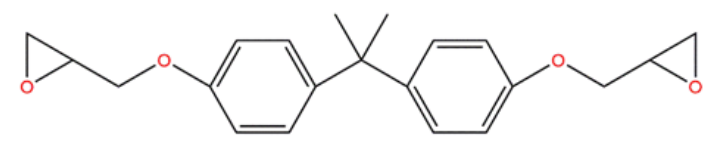

2) DGEBF<smiles>c1cc(OCC2CO2)ccc1Cc1ccc(OCC2CO2)cc1</smiles>

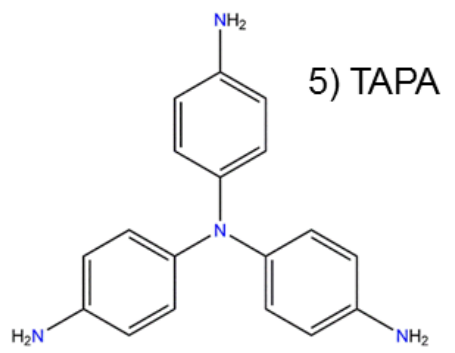

Figure 3: Molecular structures of epoxy and amine molecules studied in this work. 

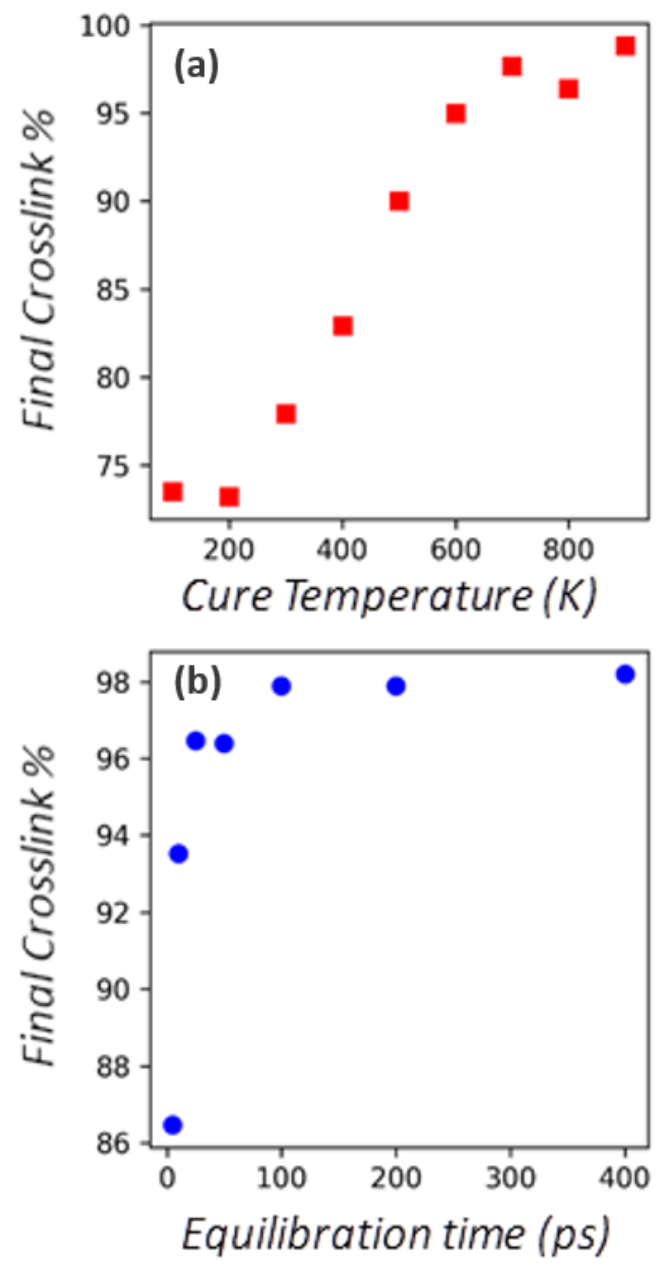

Figure 4: Effect of cure temperature and equilibration time on final crosslink saturation. DGEBA/3,3-DDS was isothermally crosslinked using Schrödinger Materials Science Suite 16-4 at (a) a range of temperatures between $100 \mathrm{~K}$ and $900 \mathrm{~K}$ with a 50ps MD equilibration step and (b) at $800 \mathrm{~K}$ with varying MD equilibration time periods. The final crosslink \% for each simulation is plotted. 

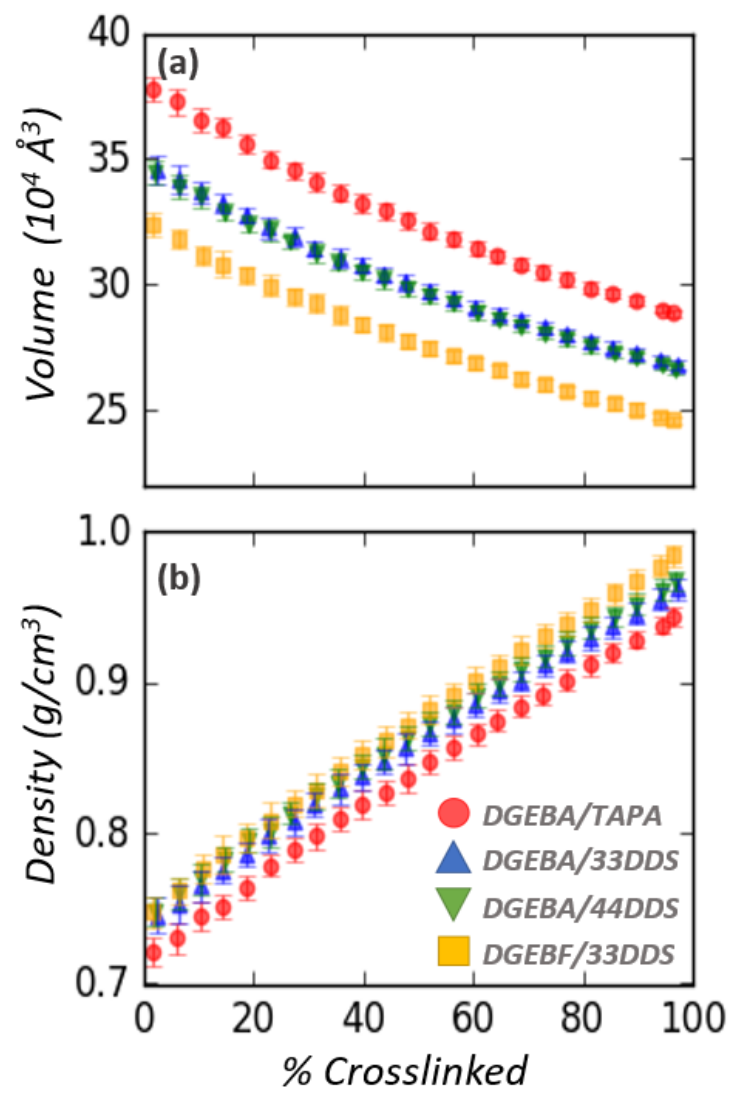

Figure 5: Evolution of physical properties during the cure cycle. The average (a) volume and (b) density during cure at $800 \mathrm{~K}$ for each of the 4 epoxy/amine systems. Error bars denote the standard deviation of ten trials and are with in the magnitude of the figure point size if not visible. 

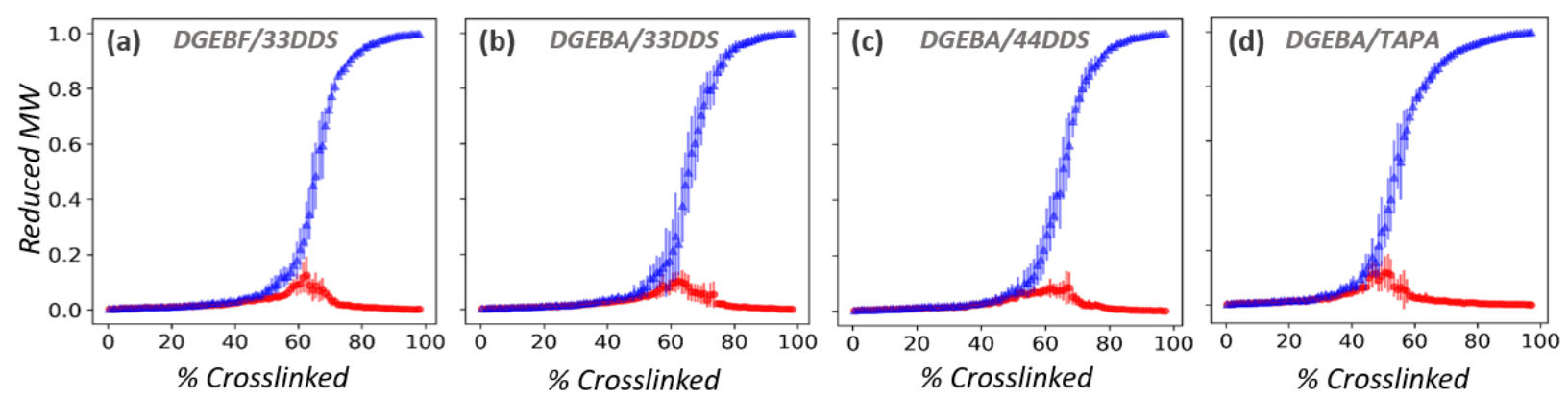

Largest MW Species

$2^{\text {nd }}$ Largest MW Species

Figure 6: Theoretical gel point estimation. The average largest and second largest MW species are plotted as a function of crosslink percentage for a) DGEBF/3,3-DDS, b) DGEBA/3,3-DDS, c) DGEBA/4,4-DDS and d) DGEBA/TAPA. Error bars denote the standard deviation of ten trials. 

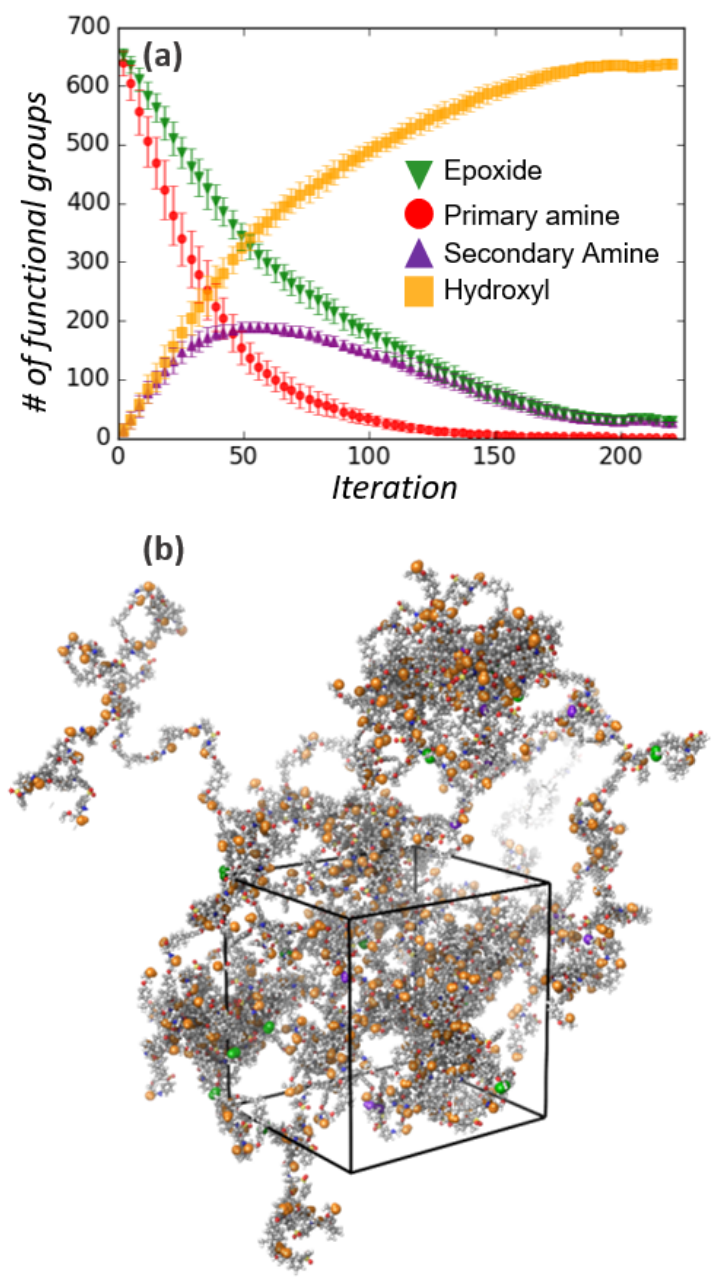

Figure 7: Tracking functional groups using SMARTS during crosslinking simulations. (a) The number of functional groups of interest are counted at each iteration of crosslinking using SMARTS patterns and averaged for 10 DGEBA/3'3'-DDS trials. Error bars denote the standard deviation of ten trials. (b) Representative snapshot from final crosslinked DGEBA/3'3'-DDS structure without periodic cell wrapping applied in visualization in order to show network connection developed during crosslinking. Functional groups are enlarged and colored to correspond to part a. 


\section{Benzoxazine Thermoset}

(a)<smiles>CC(C)(c1ccc2c(c1)CN(c1ccccc1)CO2)c1ccc2c(c1)CN(c1ccccc1)CO2</smiles>
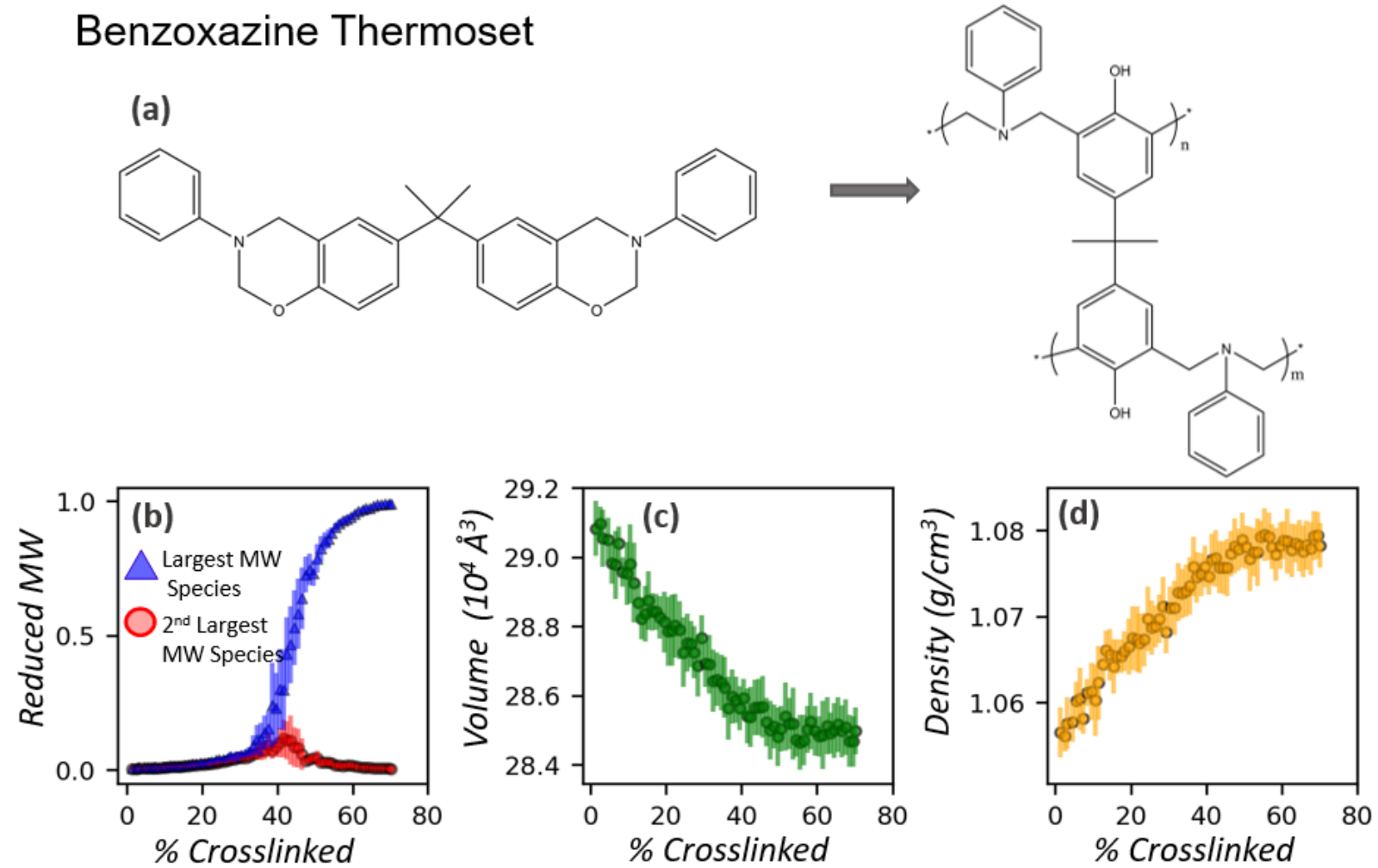

Figure 8: Benzoxazine thermoset resin cure. (a) polybenzoxazine cure reaction modeled within our simulations. (b) Estimation of benzoxazine gel point (c) Change in benzoxazine resin volume during cure at $500 \mathrm{~K}$ and (d) the change in the benzoxazine resin density during cure at $500 \mathrm{~K}$. All error bars denote the standard deviation of ten trials. 


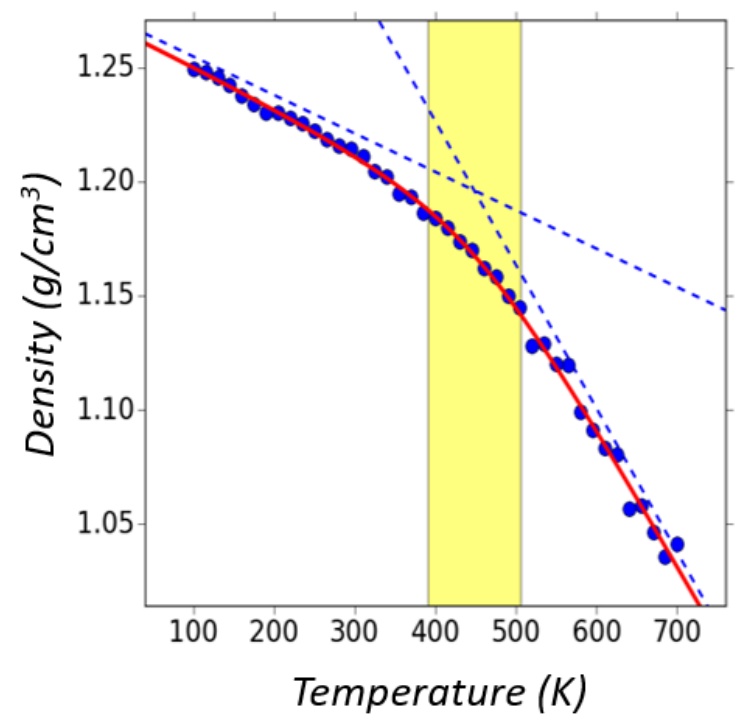

Figure 9: Predicted Glass Transition temperature. Representative data for single trial of DGEBA $/ 3^{\prime} 3^{\prime}$-DDS. The systems density as a function of temperature is fit to a hyperbolic function where the hyperbolic center is used to determine $\mathrm{T}_{\mathrm{g}}$. The asymptotic regions are denoted using blue dotted lines and the asymptotic threshold at $90 \%$ is shown in yellow. [22] 
Table 1. Properties for epoxy/amine thermoset polymers in this study.

\begin{tabular}{|c|c|c|c|c|c|c|c|c|c|c|c|c|c|}
\hline Epoxy & Amine & \# molecules & \# atoms & Cure \% & $\begin{array}{l}\text { Theoretical } \\
\text { gel point (\%) }\end{array}$ & $\begin{array}{c}\text { Sim. Gel } \\
\text { point }^{\mathrm{a}} \\
(\%)\end{array}$ & $\begin{array}{l}\Delta V^{b} \\
(\%)\end{array}$ & $\begin{array}{c}\text { CTE } \\
(300 \mathrm{~K})\end{array}$ & $\begin{array}{c}\text { CTE } \\
(600 \mathrm{~K})\end{array}$ & $\begin{array}{c}\rho^{c} \\
(300 k)\end{array}$ & $\begin{array}{c}T_{g}^{d} \\
(\exp ; K)\end{array}$ & $\begin{array}{c}\mathrm{T}_{\mathrm{g}}^{\mathrm{e}} \\
(\operatorname{sim} ; \mathrm{K})\end{array}$ & $\begin{array}{l}\operatorname{Tg~UQf~}^{\mathrm{f}} \\
(\operatorname{sim} ; \mathrm{K})\end{array}$ \\
\hline DGEBF & 3,3-DDS & 501 & 19205 & 98 & 58 & 62 & -2.5 & 163.26 & 526.38 & 1.22 & 431 & 446 & 5 \\
\hline DGEBA & 3,3-DDS & 501 & 21209 & 98 & 58 & 62 & -1.6 & 160.66 & 543.3 & 1.19 & 450 & 467 & 12 \\
\hline DGEBA & 4,4-DDS & 501 & 21209 & 97 & 58 & 55 & -2.2 & 167.3 & 570.86 & 1.17 & 473 & 480 & 26 \\
\hline DGEBA & TAPA & 500 & 23375 & 96 & 45 & 50 & -1.5 & 181.76 & 438.2 & 1.14 & 386 & 465 & 15 \\
\hline
\end{tabular}

a Gel points were estimated from the peak of the second largest MW species.

${ }^{b}$ Volume shrinkage was determined from the difference between the initial monomer and final cross-linked volume of each system at $300 \mathrm{~K}$.

${ }^{c}$ Density values reported are for the cross-linked polymer.

d Experimental Tg values references: DGEBF/3,3-DDS [23], DGEBA/3,3-DDS [13], DGEBA/4,4-DDS [10], DGEBA/TAPA [48].

${ }^{\mathrm{e}} \mathrm{T}_{\mathrm{g}}$ values were determined from hyperbola fits.

$f$

95\% confidence interval predicted using hyperbolic fit methodology [23] for 10 replicates. 
Table 2. Comparison of CTE from experiment and this study.

\begin{tabular}{cccccc}
\hline Epoxy & Amine & $\begin{array}{c}\text { Exp. Glassy linear CTE } \\
(\mathrm{exp}, \mu \mathrm{e} / \mathrm{K})^{\mathrm{a}}\end{array}$ & $\begin{array}{c}\text { Sim. Glassy } \\
\text { linear CTE } \\
(\mu \mathrm{e} / \mathrm{K})\end{array}$ & $\begin{array}{c}\text { Exp. Rubbery } \\
\text { linear CTE } \\
(\mu \mathrm{e} / \mathrm{K})^{\mathrm{a}}\end{array}$ & $\begin{array}{c}\text { Sim Rubbery } \\
\text { linear CTE } \\
(\mu \mathrm{e} / \mathrm{K})\end{array}$ \\
\hline DGEBF & $3,3-D D S$ & $57.2+/-1.0$ & $49.5+/-1.7$ & $170.8+/-2.9$ & $188.7+/-2.1$ \\
DGEBA & $3,3-D D S$ & $62.1+/-1.3$ & $50.5+/-1.8$ & $171.1+/-2.4$ & $187.1+/-4.1$ \\
DGEBA & $4,4-D D S$ & $70.0+/-0.7$ & $51.6+/-2.6$ & $163.5+/-3.3$ & $177.4+/-4.3$ \\
\hline
\end{tabular}

${ }^{\mathrm{a}}$ Experimental linear CTE values were taken from [41]. 